\title{
A Novel User Pairing Algorithm for LTE Femtocell Uplink
}

\author{
Jie Wang, Chen Xu \\ National Mobile Communications Research Laboratory, Southeast University, China \\ Email: wangjie@seu.edu.cn
}

\begin{abstract}
This paper considers the user pairing algorithm for LTE (Long Term Evolution) femtocell uplink V-MIMO (Virtual Multiple Input Multiple Output) Systems, and propose a new simulated annealing based bit error rate pairing scheduling (SA-BPS) algorithm. The SA-BPS uses Round Robin (RR) criterion to decide the first user, and simulated annealing (SA) algorithm with objective function of bit error rate (BER) is suggested to decide the pairing users. Simulation results show that the proposed SA-BPS algorithm outperforms the conventional SA algorithm in terms of BER performance. Moreover, as a growth control coefficient is used to control the growth of average BER of users in the pairing group, rapid user BER deterioration can be prevented by adopting a proper coefficient .
\end{abstract}

Index Terms- User Pairing; Femtocell; Simulated Annealing; V-MIMO; Bit Error Rate

\section{INTRODUCTION}

As the performance of V-MIMO (Virtual Multiple Input Multiple Output) system is constrained by the presence of inter-user interference, it can be enhanced by user pairing so that minimal inter-user interference can be achieved. Several user pairing strategies have been declared in [1]-[6], such as Random Pairing Scheduling (RPS), Determinant Pairing Scheduling (DPS) and Capacity Pairing Scheduling (CPS). RPS achieves low computation complexity while inter-user interference can hardly be cancelled. DPS selects pairing users according to their channel conditions, while satisfying performance can only be achieved under ideal uplink power control assumption. CPS achieves better system performs, yet its complexity rises rapidly with the increase of the number of users.

A promising user pairing strategy based on simulated annealing (SA) has been proposed in [7]. With the memory of the last transmit antenna selection step, this SA pairing algorithm can easily find the asymptotical optimal pairing user group, which achieves almost the same system performance as the greedy algorithm. However, when used in LTE femtocell, the bit error rate (BER) performance of SA pairing algorithm becomes to deteriorate, since the distance of receive antenna is limited by the mechanical size of femtocell, which raises channel correlation. As far as concerned, literatures specifically concerning user pairing algorithms of femtocell case has been rarely reported.

In this paper, a SA based BER pairing scheduling (SA-BPS) algorithm is proposed. This SA-BPS algorithm combines SA algorithm with objective function of BER. Simulation results show that, in LTE femtocell case, the proposed SA-BPS algorithm outperforms the conventional SA algorithm in terms of BER performance. Moreover, as a growth control coefficient is used to control the growth of average BER of users in the pairing group, rapid user BER deterioration can be prevented by adopting a proper coefficient .

The rest of the paper is organized as follows. In Section II, the system model is considered. Section III describes the SA-BPS algorithm. Simulation results are presented in Section IV. Finally, Section V concludes the paper.

\section{SYSTEM MODEL}

The LTE femtocell uplink V-MIMO system has $N_{R}$ receive antennas at $\mathrm{HeNB}$ (Home eNodeB or femtocell) and a single transmit antenna at each UE, We consider the $N_{T} \times N_{R}$ MIMO case, where $N_{T}$ stands for the number of users in the pairing group, while the pairing strategy will be discussed in the next Section. The transmitter and receiver models of the femtocell system under consideration are shown in Fig. 1 and Fig. 2, respectively.

On the transmitter side, the data block of each UE containing $M$ symbols is transformed by an $M$ point Fast Fourier Transform (FFT) to a frequency domain form before mapping to $N(N>M)$ orthogonal subcarriers. Then the outputs are put to an $N$ point Inverse FFT (IFFT) module to transform to time domain complex signal sequences.

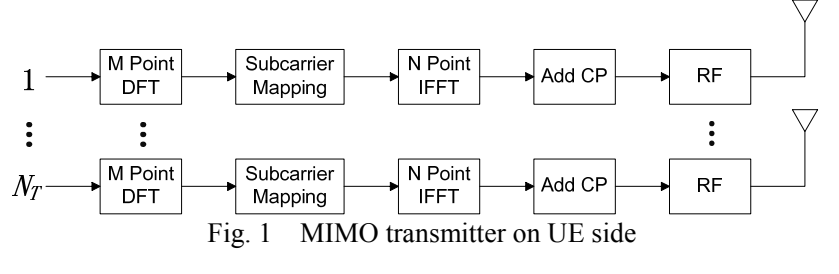

Two different approaches are available for mapping subcarriers, namely localized mapping and distributed mapping. In this paper, only the localized FDMA transmission is considered. After Circle Prefix (CP) insertion, the signal sequences are directed to the Radio Frequency (RF) module. In the channel, noise and interference are attached to the signals. On the receiver side, after signal sequences are get from the RF module, the CP will be removed first. Then an $N$ FFT will be applied to transform the signal sequences into frequency domain sequences. After subcarrier demapping, M symbols will be send to the MMSE Detector[8]. The symbol on the $m$ th $(m \in\{1, \cdots M\})$ subcarriers can be expressed as

$$
\boldsymbol{r}[m]=\boldsymbol{H}[m] \boldsymbol{s}[m]+\boldsymbol{w}[m]
$$

where 


$$
\boldsymbol{H}[m]=\left[\begin{array}{cccc}
H_{11}[m] & H_{21}[m] & \cdots & H_{N_{T} 1}[m] \\
H_{12}[m] & H_{22}[m] & \cdots & H_{N_{T} 2}[m] \\
\cdots & \cdots & \cdots & \cdots \\
H_{1 N_{R}}[m] & H_{2 N_{R}}[m] & \cdots & H_{N_{T} N_{R}}[m]
\end{array}\right]
$$

is the frequency domain channel matrix on the $m$ th subcarrier, where $H_{t r}[m]\left(1 \leq t \leq N_{T}, 1 \leq r \leq N_{R}\right)$ donates the frequency domain channel gain between the $t$ th UE antenna in the pairing group and the $r$ th receive antenna on the $m$ th subcarrier; $\boldsymbol{s}[m]=\left[s_{1}[m] s_{2}[m] \cdots s_{N_{T}}[m]\right]^{\mathcal{T}}$ donates the frequency domain data, on the $m$ th subcarrier, of the $N_{T}$ users in the pairing group; $\boldsymbol{w}[m]=\left[w_{1}[m] w_{2}[m] \cdots w_{N_{R}}[m]\right]^{\mathcal{T}} \in \mathbb{C}^{N_{R} \times 1} \quad$ is a complex Gaussian noise vector with zero mean and covariance matrix $N_{0} \boldsymbol{I} \in \mathbb{R}^{N_{R} \times N_{R}}$, i.e., $\boldsymbol{w}[m] \sim \mathcal{C N}\left(0, N_{0} \boldsymbol{I}\right)$; $\boldsymbol{r}[m]=\left[r_{1}[m] r_{2}[m] \cdots r_{N_{R}}[m]\right]^{\mathcal{T}}$ donates the frequency domain received symbol on the $m$ th subcarrier on the $N_{R}$ receive antennas.

After frequency domain received data sequences pass through the MMSE detector, the estimated frequency domain data sequence of the $N_{T}$ users in the pairing group can be obtained. According to MMSE criterion [9], the estimated frequency domain data sequence of on the $m$ th subcarrier $\hat{\boldsymbol{s}}[m]$ is given by

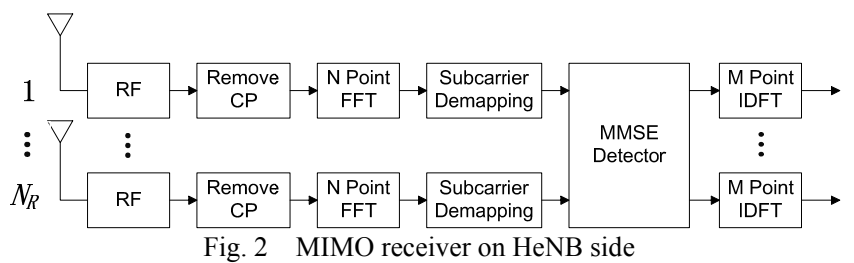

$$
\widehat{\boldsymbol{s}}[m]=\boldsymbol{A}[m] \boldsymbol{r}[m]
$$

where

$$
\boldsymbol{A}[m]=\boldsymbol{H}^{\mathcal{H}}[m]\left(\boldsymbol{H}[m] \boldsymbol{H}^{\mathcal{H}}[m]+N_{0} \boldsymbol{I}\right)^{-1}
$$

is the equalization matrix on the $m$ th subcarrier.

\section{SA-BPS ALGORITHM}

To study the pairing algorithm, we consider the femtocell uplink V-MIMO system, where HeNB, configured $N_{R}$ receive antennas, serves $N_{0}$ users, each equipped with a single transmit antenna. $N_{T}\left(N_{T} \leq N_{0}\right)$ transmit antennas are selected to form a pairing group, where $N_{T}$ is defined by the pairing strategy. All the $N_{T}$ transmit antennas transmit independent signals on the same time-frequency blocks.

In this section we concentrate on the proposed SA-BPS algorithm. This pairing algorithm combines SA algorithm with objective function of BER. The method to select the optimal antennas to form the pairing group can be formulated as

$$
\boldsymbol{Q}=\arg \min _{\boldsymbol{Q} \in \mathfrak{B}_{N_{0}}} \Delta \overline{B E R}=\arg \min _{\boldsymbol{Q} \in \mathfrak{B}_{N_{0}}} \sum_{n=1}^{N_{0}} q_{n} \Delta \overline{B E R_{n}}
$$

on condition that

$$
\sum_{n=1}^{N_{0}} q_{n} \leq N_{R}, q_{n} \in\{0,1\}
$$

and

$$
\eta(i) \leq \eta_{c}
$$

where, $\boldsymbol{Q}$ donates a $N \times 1$ pairing indication vector with the $n$th element $q_{n}$ defined as: $q_{n}=1$ if the $n$th antenna is selected to join in the pairing group, otherwise, $q_{n}=0$; $\mathfrak{B}_{N_{0}}$ donates the set of all possible pairing indication vector; $\eta_{c}$, the growth control coefficient of BER, is constant that can adopt different value so as to restrict the growth rate of average BER of users in the pairing group properly; $\triangle \overline{B E R}$ is the variation of average BER during the whole pairing procedure, which can be expressed as

$$
\Delta \overline{B E R}=\sum_{i=2}^{N_{T}} \Delta \overline{B E R(i)}
$$

where, $N_{T}$ is the number of antennas in the pairing group after the pairing procedure is done; $\triangle \overline{B E R(i)}$ donates the variation of the average BER of the users in the pairing group after the $i$ th pairing step, as is described is equation (9); $\eta(i)$ donates the growth rate of the average BER of the users in the pairing group after the $i$ th pairing step, in respect to the average BER in the $i-1$ th pairing step, as is given by equation (10).

$$
\begin{aligned}
\Delta \overline{B E R(i)} & =\overline{B E R(i)}-\overline{B E R(i-1)} \\
\eta(i) & =\frac{\Delta \overline{B E R(i)}}{\overline{B E R(i-1)}}
\end{aligned}
$$

where, $\overline{B E R(i)}$ is the average BER of the users in the pairing group after the $i$ th pairing step, given by

$$
\overline{B E R(i)}=\frac{1}{i} \sum_{j=1}^{i} B E R_{n_{j}}(i)
$$

where, $B E R_{n_{j}}(i)$ donates the BER ofthe $j$ th user from the $i$ users in the pairing group after the $i$ th pairing step [10], which can be expressed as

$$
B E R_{n_{j}}(i)=\frac{N_{\min }}{\log _{2} M_{0}} \mathcal{Q}\left(\sqrt{\xi \cdot \operatorname{SINR}_{\mathrm{n}_{j}} \cdot G_{c}}\right)
$$

where $N_{\min }$ donates the average number of nearest neighbors of a constellation point of the modulation alphabet; $M_{0}$ donates the size of the modulation alphabet; $\mathcal{Q}()$ donates $\mathrm{Q}$ function, which is defined as $\mathcal{Q}(\alpha)=$ $\int_{\alpha}^{\infty} \frac{1}{\sqrt{2 \pi}} e^{-\frac{z^{2}}{2}} d z$; the coefficient $\xi$ is defined as $\xi=$ $3 /\left[R\left(M_{0}-1\right)\right]$, where $R$ is the channel coding rate; $G_{c}$ is

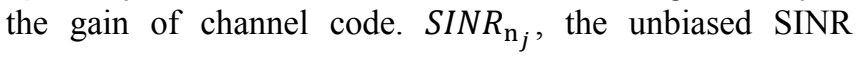
(Signal to interference plus Noise Ratio) of the $j$ th pairing user, is obtained by

$$
\operatorname{SINR}_{\mathrm{n}_{j}}=\frac{\sigma_{\mathrm{a}_{n_{j}}}^{2}}{\sigma_{e_{n_{j}}}^{2}}-1
$$

where $\sigma_{\mathrm{a}_{n_{j}}}^{2}$ is the variance of the signal of the $j$ th pairing user; $\sigma_{e_{n_{j}}}^{2}$, the error variance of the detected signal of the $j$ th pairing user, is defined as 


$$
\sigma_{e_{n_{j}}}^{2}=\frac{1}{M} \sum_{m=0}^{M-1} \Sigma_{n_{j} n_{j}}^{2}[m]
$$

where $M$ is the number of subcarriers; the intermedium variable $\Sigma_{n_{j} n_{j}}^{2}[m](1 \leq j \leq i)$ is constrained by the following equation [11]:

$$
\begin{aligned}
& {\left[\begin{array}{llll}
\Sigma_{n_{1} n_{1}}^{2}[m] & \Sigma_{n_{1} n_{2}}^{2}[m] & \cdots & \Sigma_{n_{1} n_{i}}^{2}[m] \\
\Sigma_{n_{2} n_{1}}^{2}[m] & \Sigma_{n_{2} n_{2}}^{2}[m] & \cdots & \Sigma_{n_{2} n_{i}}^{2}[m] \\
\Sigma_{n_{i} n_{1}}^{2}[m] & \Sigma_{n_{i} n_{2}}^{2}[m] & \cdots & \Sigma_{n_{i} n_{i}}^{2}[m]
\end{array}\right]} \\
& =\sigma_{n}^{2}\left(\boldsymbol{H}^{\mathcal{H}}[m] \boldsymbol{H}[m]+\frac{\sigma_{n}^{2}}{\sigma_{a_{n_{j}}}^{2}} \boldsymbol{I}_{i}\right)^{-1}
\end{aligned}
$$

where superscript $(\cdot)^{\mathcal{H}}$ denotes Hermitian transpose; $\sigma_{n}^{2}$ is the variance of noise; $\boldsymbol{I}_{i}$ donates the $i$ dimension identity matrix; $\boldsymbol{H}[m]$, the frequency domain channel equation on $m$ th subcarrier of the users in the pairing group after the $i$ th pairing step, is written as

$$
\boldsymbol{H}[m]=\left[\begin{array}{cccc}
H_{n_{1} 1}[m] & H_{n_{2} 1}[m] & \cdots & H_{n_{i} 1}[m] \\
H_{n_{1} 2}[m] & H_{n_{2} 2}[m] & \cdots & H_{n_{i} 2}[m] \\
\cdots & \cdots & \cdots & \cdots \\
H_{n_{1} N_{R}}[m] & H_{n_{2} N_{R}}[m] & \cdots & H_{n_{i} N_{R}}[m]
\end{array}\right]
$$

where $H_{n_{j} r}[m]\left(1 \leq j \leq i, 1 \leq r \leq N_{R}\right)$ donates the frequency domain channel gain between the $j$ th pairing antenna and the $r$ th receive antenna on the $m$ th subcarrier.

The procedure of the proposed SA-BPS algorithm can be summarized as follow:

(1) The 1st pairing step: Choose the first transmit antenna to join in the pairing group, based on RR, from all $N_{0}$ users served by femtocell, by setting $q_{n_{1}}=1$, where $q_{n_{1}}$ is an element of the pairing indication vector $\boldsymbol{Q}(1)$ and $n_{1}$ denotes the index of the selected antenna.

(2) The $i$ th $(i \geq 2)$ pairing step: Select another transmit antenna to join in the pairing group on the basis of $i$-1 th pairing step, by setting $q_{n_{i}}=1$, thus to make $\triangle \overline{B E R(i)}$ minimum, where $q_{n_{i}}$ is an element of the pairing indication vector $\boldsymbol{Q}(i)$ and $n_{i}$ denotes the index of the selected antenna.

(3) Compare $N_{q}$ with $N_{R}$, where $N_{q}$, the number of users already in the pairing group, is given by $N_{q}=$ $\sum_{n=1}^{N} q_{n}=\sum_{j=1}^{i} q_{n_{j}}$. If $N_{q}<N_{R}$, execute (4),otherwise (5).

(4) Compare $\eta(i)$ with $\eta_{c}$. If $\eta(i) \leq \eta_{c}$, set $i=i+1$ and execute (2) to continue the next pairing step, otherwise execute (5).

(5) The pairing procedure is accomplished, and the pairing indication vector $\boldsymbol{Q}(i)$ is the optimal solution for the pairing problem under SA-BPS algorithm, and $N_{T}=i$ is the number of the chosen receive antennas. Thus, HeNB informs all the $N_{T}$ users in the pairing group to transmit signals simultaneously at the begging of the next scheduling period.

\section{Simulation RESUlts}

In this section, the performance of the proposed SA-BPS algorithm is presented by simulation results. A single femtocell scenario is considered, where one HeNB severs several users. The HeNB is configured with two antennas while each UE equips one antenna. The users in the pairing group form a V-MIMO system with HeNB. The overall simulation parameters [12] are shown in Table I.

TABLE I. SIMULATION PARAMETERS

\begin{tabular}{|l|l|}
\hline Parameter & Value \\
\hline Channel bandwidth & $20 \mathrm{MHz}$ \\
\hline Sampling rates & $30.72 \mathrm{MHz}$ \\
\hline Modulation & QPSK \\
\hline Number of total sub-carrier & 2048 \\
\hline Number of carrier for user & 1200 \\
\hline Channel model & $3 \mathrm{GPP}$ EPA channel 7 path \\
\hline Maximum doppler frequency & $6.67 \mathrm{~Hz}$ \\
\hline Subcarrier mapping & Localized mode \\
\hline Channel codes & Turbo codes \\
\hline Channel estimation & Perfect \\
\hline BER growth control coefficient & 0.1 \\
\hline
\end{tabular}

Fig.3 shows the comparison of the BER performance of SA and SA-BPS algorithm. As is depicted in Fig.3, the proposed SA-BPS algorithm outperforms the conventional SA algorithm on BER under different signal to noise ratio (SNR) scenario. Fig.4 demonstrates that the BER gap between SA and SA-BPS algorithm will be widened, when the number of users in femtocell increases. Moreover, as a growth control coefficient is adopted in SA-BPS to control the growth of average BER of users in the pairing group, the gap will be even greater when the number of receive antenna doubles, which is not show in the figure.

\section{V.CONCLUSION}

In this paper, we propose a SA-BPS algorithm in the femtocell uplink Virtual-MIMO System. Compared with the conventional SA algorithm, the proposed pairing algorithm suits the femtocell case more properly, and achieves better BER performance. Besides, as a growth control coefficient is adopted by SA-BPS, rapid user BER deterioration can be prevented. The results can serve as plan for femtocell uplink user pairing scheduling.

\section{ACKNOWLEDGEMENT}

This work was supported by the China MIIT Municipal Science and Technology Project (2011ZX03001-006-02) and the National High Technology Research and Development Program of China (863 Program 2012AA011401). Thus the author would like to express the appreciation to their warmly support.

\section{REFERENCES}

[1] Sagar Dhakal, JoonBeom Kim, "Statistical Analysis of User-pairing Algorithms in Virtual MIMO Systems", Wireless Telecommun. Symposium, 2010, P1-5.

[2] Juan HAN, Xiaofeng TAO, Qimei CUI, "Simplified SINR-based User 
Pairing Scheduling for Virtual MIMO", Vehicular Technology Conf., 2009, P1-4.

[3] Xia Chen, Honglin Hu, Haifeng Wang, Hsiao-Hwa Chen, "Double Proportional Fair User Pairing Algorithm for Uplink Virtual MIMO

[5] Emanuele Viterbo, Ari Hottinen, "Optimal user pairing for multiuser MIMO", Spread Spectrum Techniques and Applications, 2008, P242-246.

[6] C. Wang, Q. Cui, S. Li, X. Tao, X. Xu, "Multiuser Pairing in Uplink CoMP MU-MIMO Systems Using Particle Swarm Optimization", Vehicular Technology Conf., 2011, P1-5.

[7] Bin Fan, Wenbo Wang, Yicheng Lin, Lin Huang, Kan Zheng, "Spatial Multi-user Pairing for Uplink Virtual-MIMO Systems with Linear Receiver", Wireless Commun. and Networking Conf., 2009, P1-5.

[8] Zihuai Lin, Pei Xiao, Branka Vucetic, and Mathini Sellathurai, "Analysis of Receive Algorithms for LTE SC-FDMA Based Uplink MIMO Systems" Wireless Commun. IEEE Trans. Vol. 9, 2010, P60-65.

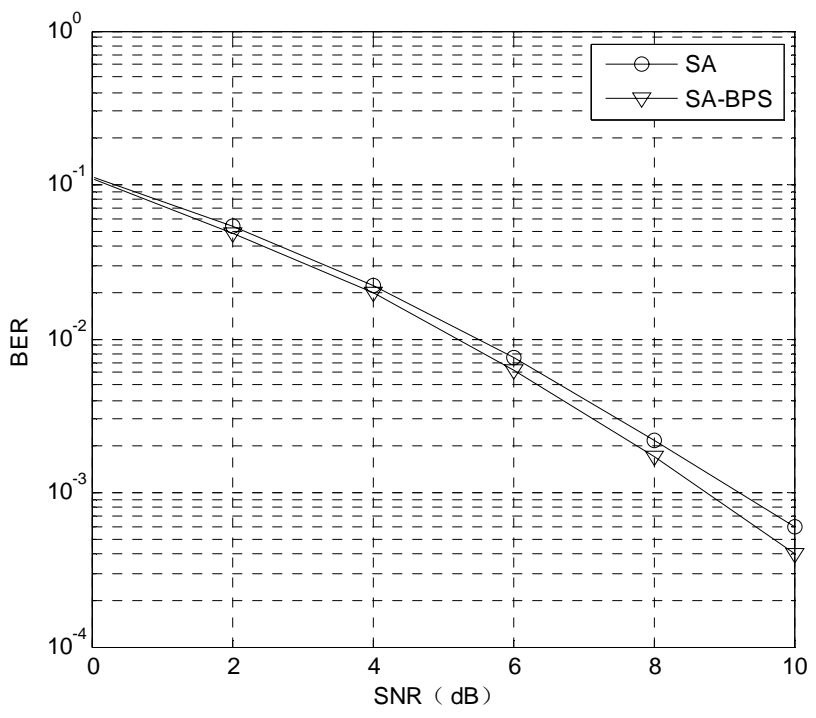

Fig. 3 BER of SA and SA-BPS algorithm, $N_{0}=4$
Systems", Wireless Commun., 2008, P2425-2429.

[4] M. A. Ruder, D. Ding, U. L. Dang, W. H. Gerstacker, “Combined User Pairing and Spectrum Allocation for Multiuser SC-FDMA Transmission", International Conf. on Commu., 2011, P1-6.

[9] William Shieh, and Ivan Djordjevic, "Orthogonal Frequency Division Multiplexing for Optical Communications[M]", London, Elsevier, 2010, P132-135.

[10] M. A. Ruder, U. L. Dang, and W. H. Gerstacker, "User Pairing for Multiuser SC-FDMA Transmission over Virtual MIMO ISI Channels", Global Telecommun. Conf., 2009, P1-7.

[11] W. H. Gerstacker, P. Nickel, F. Obernosterer, U. L. Dang, P. Gunreben, and W. Koch, "Trellis-Based Receivers for SC-FDMA Transmission over MIMO ISI Channels," in Proc. IEEE International Conference on Communications (ICC '08), May 2008, pp. 4526-4531.

[12] Yinmin Wang and Shaohui Sun, "TD-LTE Principles and System Design[M]”, Beijing, Post \& Telecom Press, 2010, P55.

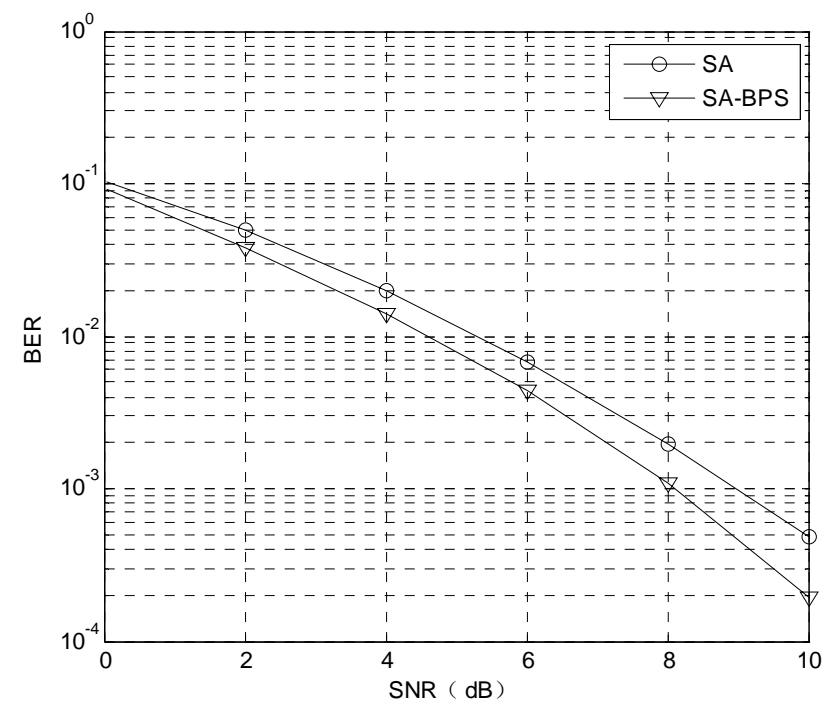

Fig.4 BER of SA and SA-BPS algorithm, $N_{0}=8$ 